\title{
Sensibilidade das manobras de Laségue e de slump nos diagnósticos de hérnia e abaulamento discal em comparação com ressonância magnética*
}

\section{Sensitivity of Laségue Sign and Slump Test in Hernia and Disc Bulging Diagnoses Compared with Magnetic Resonance Imaging}

\author{
Isabel Hahn Miranda1(1) José Luiz Pozo Raymundo ${ }^{20}$ Kevin Maahs Klein ${ }^{3(0)}$ \\ 1 Serviço de Ortopedia, Hospital Pequeno Príncipe, Curitiba, PR, Brasil \\ 2 Serviço de Ortopedia, Universidade Federal de Pelotas, Pelotas, RS, Brasil \\ ${ }^{3}$ Serviço de Cirurgia Geral, Hospital Ernesto Dornelles, Porto Alegre, \\ Endereço para correspondência Isabel Hahn Miranda, MD, \\ Universidade Federal de Pelotas (UFPel), Rua Gomes Carneiro, 01, \\ Centro, Pelotas, RS, Brasil (e-mail: isabelhmiranda@gmail.com).
} RS, Brasil

Rev Bras Ortop 2021;56(6):761-765.

\begin{abstract}
Resumo
Objetivo Mostrar a acurácia das manobras mais usadas no diagnóstico clínico da lombociatalgia, a manobra de slump e a de Laségue.

Métodos Para a realização do presente estudo, foram selecionados 101 pacientes com discopatia na ressonância magnética (RM) (padrão ouro), sendo posteriormente realizada a revisão dos prontuários para identificar quais tiveram as manobras positivas no exame físico inicial.

Resultados A sensibilidade encontrada para as manobras de slump e Laségue no diagnóstico de hérnia discal foi respectivamente $55,3 \%$ e $18,1 \%$. Já quando compara-

Palavras-chave

- sensibilidade e especificidade

- dor lombar

- hérnia discal

- radiculopatia mos as manobras para o diagnóstico de abaulamento discal, a sensibilidade obtida foi de $85,7 \%$ para a manobra de slump e de $28,6 \%$ para a manobra de Laségue.

Conclusão Foi constatado que a manobra de slump apresenta sensibilidade superior à da manobra de Laségue tanto para o diagnóstico de hérnia quanto para o de abaulamento discal quando comparadas a RM, e deveria estar mais presente na prática clínica.
\end{abstract}

Abstract

Objective To show the accuracy of the most used maneuvers in the clinical diagnosis of lumbosciatalgia, the slump test and the Laségue sign.

Trabalho realizado pelo Grupo de Ortopedia da Universidade Federal de Pelotas (UFPel) na Clínica de Ortopedia e Traumatologia Dr. José Raymundo, Pelotas, RS, Brasil. recebido

28 de Maio de 2020

aceito

17 de Setembro de 2020

Publicado on-line

Março 31, 2021
DOI https://doi.org/

10.1055/s-0040-1722590.

ISSN 0102-3616. (c) 2021. Sociedade Brasileira de Ortopedia e Traumatologia. All rights reserved.

This is an open access article published by Thieme under the terms of the Creative Commons Attribution-NonDerivative-NonCommercial-License, permitting copying and reproduction so long as the original work is given appropriate credit. Contents may not be used for commercial purposes, or adapted, remixed, transformed or built upon. (https://creativecommons.org/ licenses/by-nc-nd/4.0/)

Thieme Revinter Publicações Ltda., Rua do Matoso 170, Rio de Janeiro, RJ, CEP 20270-135, Brazil 


Methods In order to perform the present study, 101 patients with magnetic
resonance imaging (MRI) discopathy (gold standard) were selected and had their
medical records reviewed to identify which had the positive maneuvers on the initial
physical examination.
Results The sensitivity found for the slump test and the Laségue sign in the diagnosis
of disc herniation was $55.3 \%$ and $18.1 \%$, respectively. Nonetheless, when they were
compared with each other for the diagnosis of disc bulging, the sensitivity obtained
Keywords
was of $85.7 \%$ for the slump test and of $28.6 \%$ for the Laségue sign.
sensitivity and
specificity

\section{Introdução}

A lombociatalgia é definida como a dor que acomete a coluna lombar de maneira intermitente e, se irradia no trajeto do nervo ciático no membro inferior, comumente acomete até o pé. ${ }^{1}$

Dor lombar está entre as principais queixas que levam os pacientes a procurar o médico. Cerca de $70 \%$ dos adultos tem lombalgia em algum momento da vida, e em $14 \%$ desses casos a dor dura $>2$ semanas. $^{2}$ Os pacientes costumam buscar atendimento quando essa dor é recorrente, e tende a se irradiar para um, mais frequentemente, ou para ambos os membros inferiores, com sensação de queimação, choque ou parestesia nos dermátomos específicos do nervo ciático. Dentre as raízes que formam o nervo ciático, as raízes mais acometidas são L5 e S1.

A avaliação da dor e o exame físico são fundamentais para o diagnóstico e tratamento do paciente, por isso a importância de conhecer e saber aplicar as manobras que avaliam as raízes que compõem o nervo ciático, sendo as manobras de Laségue e slump o método de escolha na avaliação. Para melhor entendimento dos testes diagnósticos, é fundamental a compreensão do eixo de movimento dos corpos vertebrais, pois só assim se compreende por que ambos os testes são em flexão, uma vez que fletindo a coluna há compressão nas raízes, ${ }^{3}$ permitindo a suspeição clínica. Contudo, para que o uso dos testes no exame físico corrobore no atendimento ao paciente, é preciso saber qual a capacidade deles em identificar os indivíduos que apresentam verdadeiramente compressão de raiz nervosa por hérnia ou abaulamento nos níveis que compõem o nervo ciátio.

Uma vez que a dor seja característica e o exame físico sugira compressão radicular, podemos prosseguir a investigação com o exame de imagem mais apropriado. No caso de suspeita de compressão de raiz nervosa, a ressonância magnética (RM) é o exame mais indicado, pois se pode visualizar a raiz e assim evidenciar o diagnóstico correto por meio da visualização da imagem.

Pensando nisso, o presente estudo foi desenvolvido para estimar a sensibilidade das manobras de Laségue e slump, usando a RM como padrão ouro comparativo.

\section{Material e Métodos}

Para a realização do presente estudo, foram selecionados aleatoriamente 130 prontuários de pacientes em acompanhamento de dor lombar com irradiação para membro(os) inferior(es). Essa dor foi definida como dor localizada no dorso em topografia lombar que se irradiava para algum dos membros inferiores ou para ambos nos dermátomos do nervo ciático. O acompanhamento de pacientes com essa queixa é padronizado pelos autores em todos os seus atendimentos de rotina, sendo realizados anamnese e exame físico em toda primeira consulta. Esta avaliação semiológica inclui a realização das manobras de slump e de Laségue, e é seguida da realização de exame de imagem sempre que a queixa condiz com diagnóstico de lombociatalgia, sendo o exame de escolha nestes casos a RM da coluna lombar (padrão-ouro). Dos 130 selecionados inicialmente, 29 foram excluídos durante a avaliação por não preencherem o critério da RM com diagnósticos ou de hérnia ou de abaulamento, enquanto 101 pacientes foram incluídos por apresentarem essas alterações no exame de imagem.

O estudo compara a sensibilidade das manobras de slump e de Laségue no diagnóstico de hérnia e de abaulamento dos discos intervertebrais causando a dor no trajeto do nervo ciático; por esse motivo, foram selecionados apenas os pacientes que apresentavam algum desses diagnósticos no laudo da RM para que, desse modo, se pudesse analisar os prontuários de maneira retrospectiva, comparando o diagnóstico apresentado na RM com a presença ou não de positividade em cada manobra citada.

A manobra de slump foi realizada com o paciente sentado na maca, com as pernas pendentes e as mãos sobre as coxas. 0 examinador realizou a flexão do tronco sobre as coxas e a flexão do segmento cervical, aproximando o queixo ao esterno; na sequência, com a outra mão, segurou a face plantar de um dos pés e realizou a extensão do joelho e flexão dorsal do pé, mantendo a outra perna pendente e, depois, realizou o mesmo com o membro inferior contralateral. ${ }^{4}$

Já a manobra de Laségue, também aplicada bilateralmente, foi realizada com o paciente em decúbito dorsal, com os membros inferiores estendidos sobre a maca. 0 examinador, colocando uma mão em um dos calcanhares do paciente, 
realiza a flexão de até $60^{\circ}$ da coxa, mantendo o joelho estendido, em um membro de cada vez.,

Ambas as manobras foram consideradas positivas somente quando, durante sua realização, o paciente referisse dor irradiada no membro inferior que se manifestasse no trajeto do nervo ciático.

Quanto às RMs, todas foram realizadas no mesmo local e na mesma máquina, sendo todos os exames analisados pelo mesmo radiologista. Os laudos dos exames de todos os selecionados ou possuíam hérnia ou abaulamento discal.

Em referência à definição de hérnia e abaulamento, foi realizada uma revisão de literatura e, após uma análise de diversos artigos, os autores optaram pelas definições encontradas no trabalho de Jensen et al., ${ }^{7}$ no qual os autores diferenciam hérnia de abaulamento. Neste estudo, Jensen et al. diferenciaram a hérnia como podendo ser protrusão ou extrusão do núcleo pulposo; no primeiro caso, a base da ruptura é menor que o maior diâmetro do núcleo pulposo exteriorizado; já no segundo caso, a base da ruptura é maior que o maior diâmetro do núcleo pulposo exteriorizado para fora do anel fibroso. No entanto, aqui os autores considerarão apenas o diagnóstico de hérnia, sem diferenciar os subtipos de Jensen et al. Já a definição de abaulamento é descrita por Jensen et al. como uma distensão do disco no plano transverso comprimindo a raiz nervosa.

\section{Resultados}

Dos 101 pacientes incluídos, 62 (61,4\%) eram do sexo feminino, e 39 (38,6\%) eram do sexo masculino, com faixa etária variando de 18 a 89 anos; destes, 94 (93,1\%) apresentaram diagnóstico de hérnia, e 7 (6,9\%) de abaulamento no exame padrão-ouro, o que sugere maior prevalência de diagnósticos em pacientes com hérnia (- Tabela $\mathbf{1}$ ).

Durante a revisão de prontuários, constatou-se que desses 101 pacientes, 58 apresentaram a manobra de slump positiva, sendo 40 à direita, 36 à esquerda, e 18 bilaterais, enquanto 19 apresentaram a manobra de Laségue positiva, sendo 13 à direita, 9 à esquerda e 3 bilaterais. A partir disso, foi possível realizar o cálculo da sensibilidade de cada manobra nos diagnósticos de hérnia e de abaulamento dos discos intervertebrais.

A fim de avaliar a sensibilidade da manobra de slump no diagnóstico de hérnia de disco, comparou-se o número de diagnósticos de hérnia discal nas ressonâncias (94) com o

Tabela 1 Divisão por gênero dos pacientes com discopatia

\begin{tabular}{|c|l|l|l|}
\hline Variável & $\begin{array}{l}\text { Geral } \\
(\boldsymbol{n}=\mathbf{1 0 1 )}\end{array}$ & $\begin{array}{l}\text { Sexo } \\
\text { feminino } \\
(\boldsymbol{n}=\mathbf{6 2})\end{array}$ & $\begin{array}{l}\text { Sexo } \\
\text { masculino } \\
(\boldsymbol{n}=39)\end{array}$ \\
\hline Idade (anos) & $\begin{array}{l}53,2 \pm 15,7 \\
(18-86)\end{array}$ & $\begin{array}{l}53,8 \pm 16,3 \\
(18-84)\end{array}$ & $\begin{array}{l}52,1 \pm 14,9 \\
(18-89)\end{array}$ \\
\hline Discopatia & & & \\
\hline -Hérnia & $94(93,1 \%)$ & $56(90,3 \%)$ & $38(97,4 \%)$ \\
\hline - Abaulamento & $7(6,9 \%)$ & $6(9,7 \%)$ & $1(2,6 \%)$ \\
\hline
\end{tabular}

Resultados descritos por média \pm desvio padrão (mínimo - máximo) ou por frequência (percentual). número de pacientes que possuíam a manobra de slump positiva (52); portanto, se 94 equivalem a $100 \%$ das hérnias, os 52 que possuíam a manobra positiva equivalem a $55,3 \%$, sendo essa a sensibilidade da manobra de slump no diagnóstico de hérnia discal. Para avaliar a sensibilidade da manobra de Laségue, comparou-se o número de diagnósticos de hérnia nas RMs (94) com o número de pacientes que possuíam a manobra de Laségue positiva (17); portanto, se 94 equivalem a $100 \%$ das hérnias, os 17 que possuíam a manobra positiva equivalem a $18,1 \%$, sendo essa a sensibilidade da manobra de Laségue no diagnóstico de hérnia discal (-Tabela 2).

Para calcular a sensibilidade da manobra de slump no diagnóstico de abaulamento discal, comparou-se o número de diagnósticos de abaulamento discal nas RMs (7) com o número de pacientes que possuíam a manobra de slump positiva (6); portanto, se 7 equivalem a 100\% dos abaulamentos, os 6 que possuíam a manobra positiva equivalem a $85,7 \%$, sendo essa a sensibilidade dessa manobra no diagnóstico de abaulamento discal. Para calcular a sensibilidade da manobra de Laségue no diagnóstico de abaulamento discal, comparou-se o número de diagnósticos de abaulamento discal nas RMs (7) com o número de pacientes que possuíam a manobra de Laségue positiva (2); portanto, se 7 equivalem a $100 \%$ dos abaulamentos, os 2 que possuíam a manobra positiva equivalem a $28,6 \%$, sendo essa a sensibilidade dessa manobra no diagnóstico de abaulamento discal (-Tabela 3).

\section{Discussão}

No presente estudo, verificou-se que os testes avaliados apresentam diferença de sensibilidade significativa, tendo a manobra de slump uma capacidade muito maior que a manobra de Laségue de detectar os pacientes que verdadeiramente apresentam hérnia ou abaulamento discal quando comparados ao exame padrão-ouro. Esse resultado encontrado reforça os achados de Majlesi et al., ${ }^{8}$ que também encontraram diferença estatística na sensibilidade das manobras, mostrando também a maior eficácia da manobra de slump ao identificar os verdadeiros positivos.

Contudo, vale lembrar que essas manobras são testes de exame físico e, por esse motivo, podem variar um pouco de examinador para examinador e, também, conforme o limiar de dor de cada paciente. Ainda se ressalta aqui que ambas as manobras, nesse caso principalmente a de slump, podem apresentar-se falsamente positivas em pacientes que são portadores de encurtamento de isquiotibiais. Por isso, é importante que o médico tenha capacidade de reconhecer bem os dermátomos do nervo ciátio, sabendo então diferenciar as causas da dor que se manifesta posteriormente nos membros inferiores.

Sob este cenário, sugere-se que ambas as manobras sejam realizadas na busca do diagnóstico mais preciso, e valoriza-se aqui a manobra de slump, já que sua aplicabilidade traz mais segurança para confirmar a suspeita de hérnia ou abaulamento discal a nível das raízes do nervo isquiático. Ressaltase ainda que são necessários mais estudos para que o 
764 Sensibilidade das manobras de Laségue e de slump nos diagnósticos de hérnia Miranda et al.

Tabela 2 Sensibilidade das manobras de slump e Lasége no diagnóstico de hérnia discal

\begin{tabular}{|c|c|c|c|c|c|}
\hline & Manobra & Lado & Casos com alteração & Sensibili-dade & IC95\% \\
\hline \multirow[t]{8}{*}{ Geral $(n=94)$} & \multirow[t]{4}{*}{ Slump } & Direita & 34 & $36,2 \%$ & $26,5 \%-45,9 \%$ \\
\hline & & Esquerda & 35 & $37,2 \%$ & $27,5 \%-47 \%$ \\
\hline & & Bilateral & 17 & $18,1 \%$ & $10,3 \%-25,9 \%$ \\
\hline & & Dir e/ou esq & 52 & $55,3 \%$ & $45,3 \%-65,4 \%$ \\
\hline & \multirow[t]{4}{*}{ Lasègue } & Direita & 11 & $11,7 \%$ & $5,2 \%-18,2 \%$ \\
\hline & & Esquerda & 9 & $9,6 \%$ & $3,6 \%-15,5 \%$ \\
\hline & & Bilateral & 3 & $3,2 \%$ & $0 \%-6,7 \%$ \\
\hline & & Dir e/ou esq & 17 & $18,1 \%$ & $10,3 \%-25,9 \%$ \\
\hline \multirow[t]{8}{*}{ Feminino $(n=56)$} & \multirow[t]{4}{*}{ Slump } & Direita & 18 & $32,1 \%$ & $19,9 \%-44,4 \%$ \\
\hline & & Esquerda & 18 & $32,1 \%$ & $19,9 \%-44,4 \%$ \\
\hline & & Bilateral & 9 & $16,1 \%$ & $6,5 \%-25,7 \%$ \\
\hline & & Dir e/ou esq & 27 & $48,2 \%$ & $35,1 \%-61,3 \%$ \\
\hline & \multirow[t]{4}{*}{ Lasègue } & Direita & 6 & $10,7 \%$ & $2,6 \%-18,8 \%$ \\
\hline & & Esquerda & 5 & $8,9 \%$ & $1,5 \%-16,4 \%$ \\
\hline & & Bilateral & 2 & $3,6 \%$ & $0 \%-8,4 \%$ \\
\hline & & Dir e/ou esq & 9 & $16,1 \%$ & $6,5 \%-25,7 \%$ \\
\hline \multirow[t]{8}{*}{ Masculino $(n=38)$} & \multirow[t]{4}{*}{ Slump } & Direita & 16 & $42,1 \%$ & $26,4 \%-57,8 \%$ \\
\hline & & Esquerda & 17 & $44,7 \%$ & $28,9 \%-60,5 \%$ \\
\hline & & Bilateral & 8 & $21,1 \%$ & $8,1 \%-34 \%$ \\
\hline & & Dir e/ou esq & 25 & $65,8 \%$ & $50,7 \%-80,9 \%$ \\
\hline & \multirow[t]{4}{*}{ Lasègue } & Direita & 5 & $13,2 \%$ & $2,4 \%-23,9 \%$ \\
\hline & & Esquerda & 4 & $10,5 \%$ & $0,8 \%-20,3 \%$ \\
\hline & & Bilateral & 1 & $2,6 \%$ & $0 \%-7,7 \%$ \\
\hline & & Dir e/ou esq & 8 & $21,1 \%$ & $8,1 \%-34 \%$ \\
\hline
\end{tabular}

Abreviações: IC, intervalo de confiança.

Tabela 3 Sensibilidade das manobras de slump e Laségue no diagnóstico de abaulamento discal

\begin{tabular}{|c|c|c|c|c|}
\hline & Manobra & Lado & Casos com alteração & Sensibilidade \\
\hline \multirow[t]{8}{*}{ Geral $(n=7)$} & \multirow[t]{4}{*}{ Slump } & Direita & 6 & $85,7 \%$ \\
\hline & & Esquerda & 1 & $14,3 \%$ \\
\hline & & Bilateral & 1 & $14,3 \%$ \\
\hline & & Dir e/ou esq & 6 & $85,7 \%$ \\
\hline & \multirow[t]{4}{*}{ Lasègue } & Direita & 2 & $28,6 \%$ \\
\hline & & Esquerda & 0 & $0 \%$ \\
\hline & & Bilateral & 0 & $0 \%$ \\
\hline & & Dir e/ou esq & 2 & $28,6 \%$ \\
\hline \multirow[t]{6}{*}{ Feminino $(n=6)$} & \multirow[t]{4}{*}{ Slump } & Direita & 6 & $100 \%$ \\
\hline & & Esquerda & 1 & $16,7 \%$ \\
\hline & & Bilateral & 1 & $16,7 \%$ \\
\hline & & Dir e/ou esq & 6 & $100 \%$ \\
\hline & \multirow[t]{2}{*}{ Lasègue } & Direita & 2 & $33,3 \%$ \\
\hline & & Esquerda & 0 & $0 \%$ \\
\hline
\end{tabular}


Tabela 3 (Continued)

\begin{tabular}{|c|c|c|c|c|}
\hline & Manobra & Lado & Casos com alteração & Sensibilidade \\
\hline & & Bilateral & 0 & $0 \%$ \\
\hline & & Dir e/ou esq & 2 & $33,3 \%$ \\
\hline \multirow[t]{8}{*}{ Masculino $(n=1)$} & \multirow[t]{4}{*}{ Slump } & Direita & 0 & \\
\hline & & Esquerda & 0 & \\
\hline & & Bilateral & 0 & \\
\hline & & Dir e/ou esq & 0 & \\
\hline & \multirow[t]{4}{*}{ Lasègue } & Direita & 0 & \\
\hline & & Esquerda & 0 & \\
\hline & & Bilateral & 0 & \\
\hline & & Dir e/ou esq & 0 & \\
\hline
\end{tabular}

diagnóstico clínico, baseado em anamnese e exame físico, seja cada vez mais eficaz, aproximando o médico do paciente através da propedêutica, por vezes esquecida nos dias atuais.

\section{Conclusões}

Com o presente trabalho, tentou-se transmitir à comunidade cientifica que o exame físico ainda tem grande valia no diagnóstico, em especial quando o examinador conhece as diversas manobras e compreende seu valor na suspeita da patologia.

No caso das manobras de slump e Laségue, concluiu-se, por meio de um estudo com cerca de 100 pacientes, que nenhuma delas tem alta sensibilidade, porém a primeira tem uma capacidade muito maior que a segunda de sugerir o diagnóstico de lombociatalgia nos pacientes que realmente apresentam a compressão radicular evidenciada como hérnia ou abaulamento nas RMs.

\section{Declaração de Consentimento}

Eu, José Luiz Pozo Raymundo, autorizo o uso dos prontuários da minha clínica para realização de trabalho científico de revisão sem a divulgação da identidade dos pacientes por mim tratados.
Conflito de Interesses

Os autores declaram não haver conflito de interesses.

\section{Referências}

1 Albuquerque AV. Lombalgia crônica sem ciatalgia: correlação entre o quadro clínico e a radiologia. Rev Neurociênc 2008;16 (03):184-188

2 Deyo RA, Rainville J, Kent DL. What can the history and physical examination tell us about low back pain? JAMA 1992;268(06): 760-765

3 Charnley J. Orthopaedic signs in the diagnosis of disc protrusion. With special reference to the straight-leg-raising test. Lancet 1951;1(6648):186-192

4 Maitland GD. The slump test: examination and treatment. Aust J Physiother 1985;31(06):215-219

5 Sunderland S, Bradley KC. Stress-Strain Phenomena in Human Spinal Nerve Roots. Brain 1961;84(01):120-124

6 Sunderland S, Bradley KC. Stress-Strain Phenomena in Denervated Peripheral Nerve Trunks. Brain 1961;84(01):125-127

7 Jensen MC, Brant-Zawadzki MN, Obuchowski N, Modic MT, Malkasian D, Ross JS. Magnetic resonance imaging of the lumbar spine in people without back pain. N Engl J Med 1994;331(02): 69-73

8 Majlesi J, Togay H, Unalan H, Toprak S. The sensitivity and specificity of the Slump and the Straight Leg Raising tests in patients with lumbar disc herniation. J Clin Rheumatol 2008;14 (02):87-91 